\title{
Ultrasound-guided angioplasty of dialysis fistulas in renal transplant patients
}

\author{
Dariusz Szewczyk, Piotr Andziak, Krzysztof Bojakowski, Rafał Góra, Maciej Gaciong \\ $2^{\text {nd }}$ Department of Vascular Surgery, Centre of Postgraduate Medical Education, Warsaw, Poland
}

Videosurgery Miniinv 2019; 14 (4): 532-537

DOI: https://doi.org/10.5114/wiitm.2019.83316

\begin{abstract}
Introduction: Percutaneous endovascular angioplasty has become the treatment of choice for dialysis fistula stenosis. The ultrasound-guided endovascular procedure is used in patients with severe renal impairment and advanced renal transplant failure, when the need for nephrotoxic contrast administration in standard angioplasty may worsen renal function.

Aim: To evaluate endovascular angioplasty guided by ultrasound for dialysis fistula stenosis in renal transplant patients with severe graft insufficiency.

Material and methods: We compared ultrasound (US)-guided angioplasty, performed in patients after renal transplantation, with standard contrast angioplasty performed in dialysis patients. We treated 10 kidney allograft recipients (9 kidneys and 1 kidney-pancreas) with significantly compromised renal transplant function and significant stenosis in dialysis fistulas, as detected during US examination. Patients were qualified for percutaneous angioplasty under US guidance. The mean period from transplantation was 32.7 months (5-100 months). Results of their treatment were compared to the control group of 20 end-stage renal disease patients with dialysis fistula stenosis treated by angioplasty under standard contrast visualization.

Results: The immediate effectiveness of the angioplasty was 100\% in both groups. No early complications of angioplasty or problems with the guidewire crossing the stenosis were observed. Twelve months of primary patency was observed in $80 \%$ and $45 \%$ in the US-guided and control groups, respectively.

Conclusions: The US-guided endovascular procedure is an effective and safe method of treating dialysis fistula stenosis in patients with impaired renal transplant function.
\end{abstract}

Key words: dialysis fistula, angioplasty, ultrasound, renal transplant.

\section{Introduction}

Despite significant and constant improvements in renal transplant outcomes, the chance of a renal transplant patient returning to dialysis due to a graft failure is significant and reaches approximately $30 \%$ within 10 years after the transplantation [1]. Well-functioning vascular access in patients returning to haemodialysis due to renal transplant failure is necessary, but sometimes it becomes extremely difficult and is not always feasible [2]. Current data show that most patients with end-stage renal transplant insufficiency are qualified for haemodialysis and more frequently use dialysis catheters than patients who are starting dialysis [1].

The possibility of performing open redo procedures for dialysis fistulas to re-establish their proper function in patients with renal allograft insufficiency has been previously described [3, 4]. Unfortunately, standard endovascular correction of dialysis fistula stenosis, which has become the recommended

\section{Address for correspondence}

Dariusz Szewczyk MD, $2^{\text {nd }}$ Department of Vascular Surgery, Centre of Postgraduate Medical Education, St Wołoska 137, 02-507 Warsaw, Poland, e-mail: dszew@poczta.onet.pl 
method, cannot be used in non-dialyzed patients with compromised renal function due to the contrast nephrotoxicity. Ultrasound (US) visualization enables angioplasty of a dialysis fistula stenosis without nephrotoxic contrast use and can be performed in dialyzed and pre-dialyzed patients, as described previously $[5,6]$. We studied the effect of endovascular angioplasty guided by US for dialysis fistula stenosis in renal transplant patients with severe graft insufficiency. Additionally, we compared these results with angioplasty performed in dialyzed patients performed under well-established radiological control.

\section{Aim}

Aim of the study was to evaluate endovascular angioplasty guided by ultrasound for dialysis fistula stenosis in renal transplant patients with severe graft insufficiency.

\section{Material and methods}

\section{Ethics statement}

This study was approved by the Ethical Committee of the Central Clinical Hospital Ministry of Internal Affairs (35/2010). Written informed consent was obtained from all patients.

\section{Patients}

We treated 10 kidney allograft recipients (9 kidney and 1 kidney-pancreas) with significantly compromised renal transplant function and significant stenosis in dialysis fistulas, as detected during US examination. Patients were qualified for percutaneous angioplasty under US guidance. The mean period from transplantation was 32.7 months (5-100 months). Results of their treatment were compared to a control group of 20 end-stage renal disease (ESRD) patients with dialysis fistula stenosis treated by angioplasty under standard contrast visualization. Patients' characteristics are shown in Table I.

\section{Fistula}

US examination of dialysis fistula $(11 \mathrm{~L}$ linear probe, GE LogiQ GE HealthCare, USA) was performed before and after angioplasty. Localization, degree of stenosis, minimal fistula diameter and blood flow characteristics were noted. Stenosis adjacent to the arterial anastomosis, in a dialysis fistula seg- ment not used for puncturing, was defined as inflow stenosis. Stenosis in the fistula segment used for puncturing was referred to as cannulation segment stenosis. Stenosis compromising fistula flow but situated in veins located proximally to the segment commonly used for cannulation was described as outflow stenosis. Dialysis fistula characteristics are shown in Table II.

\section{US-guided angioplasty procedure}

Under US guidance, dialysis fistulas were punctured, preferably antegradely, towards the detected stenoses. A 5-6 Fr vascular sheath was introduced, followed by the hydrophilic leader $(180 \mathrm{~cm}$, HydroSteer, St. Jude Medical, USA). The diameter and length of the balloon used for the intervention were

Table I. Patient characteristics

\begin{tabular}{|c|c|c|}
\hline Characteristics & $\begin{array}{l}\text { Contrast } \\
\text { guidance }\end{array}$ & US guidance \\
\hline Sex (women/men) & $8 / 12$ & $5 / 5$ \\
\hline Age, mean (range) [years] & $64.1(22-88)$ & $45.4(30-66)$ \\
\hline Hypertension (\%) & 95 & 100 \\
\hline Diabetes mellitus (\%) & 65 & 20 \\
\hline Coronary disease (\%) & 50 & 20 \\
\hline $\begin{array}{l}\text { Coronary artery intervention } \\
(\mathrm{PTCA}+\mathrm{CABG})(\%)\end{array}$ & 20 & 0 \\
\hline Arrhythmia (\%) & 15 & 20 \\
\hline Peripheral artery disease (\%) & 30 & 0 \\
\hline$\beta$-Blocker (\%) & 90 & 80 \\
\hline Diuretic (\%) & 70 & 80 \\
\hline Ca blocker (\%) & 45 & 30 \\
\hline ACE inhibitor (\%) & 40 & 10 \\
\hline Anti-platelet (\%) & 60 & 70 \\
\hline Insulin (\%) & 45 & 0 \\
\hline Oral hypoglycaemic (\%) & 5 & 0 \\
\hline Statin (\%) & 65 & 80 \\
\hline Steroid (\%) & 25 & 90 \\
\hline Azathioprine (\%) & 0 & 10 \\
\hline Mycophenolate mofetil (\%) & 0 & 60 \\
\hline Tacrolimus (\%) & 0 & 80 \\
\hline Cyclosporin A (\%) & 0 & 20 \\
\hline
\end{tabular}


Table II. Dialysis fistula characteristics

\begin{tabular}{|lcc|}
\hline Dialysis fistula characteristics & $\begin{array}{c}\text { US } \\
\text { guidance }\end{array}$ & $\begin{array}{c}\text { Contrast } \\
\text { guidance }\end{array}$ \\
\hline Forearm/arm fistula (\%) & $30 / 70$ & $5 / 90$ \\
\hline Radio-cephalic (\%) & 30 & 5 \\
\hline Brachio-cephalic (\%) & 50 & 45 \\
\hline Brachio-basilic (\%) & 20 & 20 \\
\hline PTFE + basilic vein (\%) & 0 & 25 \\
\hline Femoral fistula (VSM) (\%) & 0 & 5 \\
\hline Stenosis severity (\%) & 59.4 & 58.3 \\
\hline Inflow stenosis (\%) & 10 & 15 \\
\hline Cannulation segment stenosis (\%) & 0 & 40 \\
\hline Outflow stenosis (\%) & 90 & 45 \\
\hline
\end{tabular}

based on the US image of the lesion. The balloon was then advanced into the stenosis. To avoid a too deep or uncontrolled leader insertion, which can lead to central vein damage, we used X-rays. Before the angioplasty, intravenous heparin (50 U/kg BW) was given. Angioplasty was performed under US guidance and, in some cases, with the help of radiological imaging during the deployment of the bal-

Table III. Results of endovascular angioplasty of dialysis fistula stenosis

\begin{tabular}{|lcc|}
\hline Parameter & US guidance & $\begin{array}{l}\text { Contrast } \\
\text { guidance }\end{array}$ \\
\hline Stenosis before PTA (\%) & $59.4 \pm 10.17$ & $58.3 \pm 1.59$ \\
\hline Stenosis after PTA (\%) & $16.8 \pm 9.19$ & $12.2 \pm 8.82$ \\
\hline $\begin{array}{l}\text { Minimal dialysis fistula } \\
\text { diameter in stenosis before } \\
\text { PTA [mm] }\end{array}$ & $3.79 \pm 1.21$ & $2.7 \pm 0.96$ \\
\hline $\begin{array}{l}\text { Minimal dialysis fistula } \\
\text { diameter in stenosis after } \\
\text { PTA [mm] }\end{array}$ & $7.73 \pm 2.26$ & $5.56 \pm 1.12$ \\
\hline $\begin{array}{l}\text { Dialysis fistula blood flow } \\
\text { before PTA [ml/min] }\end{array}$ & $868.8 \pm 392.4$ & $439.9 \pm 222.8$ \\
\hline $\begin{array}{l}\text { Dialysis fistula blood flow } \\
\text { after PTA [ml/min] }\end{array}$ & $1228.8 \pm 538.3$ & $676.4 \pm 370.5$ \\
\hline $\begin{array}{l}\text { Maximum blood velocity } \\
\text { before PTA [cm/s] }\end{array}$ & $317.8 \pm 62.3$ & $314.0 \pm 83.5$ \\
\hline $\begin{array}{l}\text { Maximum blood velocity } \\
\text { after PTA [cm/s] }\end{array}$ & $166.5 \pm 29.0$ & $167.7 \pm 54.1$ \\
\hline
\end{tabular}

Variables presented as means and standard deviations; PTA - percutaneous transluminal angioplasty. loon filled with contrast (REEF, Invatec, Italy), inflated for $3 \mathrm{~min}$ up to the rated burst pressure (> $20 \mathrm{~atm}$ ). After angioplasty, the balloon was removed and the effectiveness of the intervention was assessed in a postprocedural US examination. No contrasts were used in these patients to perform angiography.

\section{Standard angiography-controlled angioplasty}

In the control group, we performed standard angioplasty procedures. Angioplasty under radiological control did not differ from procedures performed in the US-guided group (the same type of balloon, pressure and time of PTA). Vascular access was obtained through a dialysis fistula puncture, and the angiography was performed before the procedure for the stenosis visualization and after angioplasty for post-procedural evaluation.

\section{Statistical analysis}

Statistical analyses were done using Statistica 7.0 (StatSoft). Two-group independent comparisons were done with the Mann-Whitney non-parametric test. Statistical significance was considered at $p<0.05$.

\section{Results}

We compared the results of endovascular angioplasty of dialysis fistula stenosis guided by US in renal transplant patients with severe graft insufficiency with standard procedure angioplasty in dialyzed patients under radiological control.

The minimal dialysis fistula diameter in stenosis and blood flow measured before and after angioplasty are shown in Table III. In both groups, angioplasty was an efficient method for the dilatation of dialysis fistula stenosis. Increases in the minimal fistula diameters by angioplasty in the US-guided and the control group were $2.23 \pm 0.60$ and $2.08 \pm 0.27$ times, respectively $(p>0.05)$. Decreases in maximum blood velocity after angioplasty were similar in both groups (mean drop: $46.7 \%$ in controls, and $46.5 \%$ in the US-guided group, NS). Angioplasty increased dialysis fistula blood flow by $68 \%$ in the control group and $44 \%$ in the US-guided group ( $p=0.093$ ). The percentage of patients maintaining primary patency for 12 months after angioplasty was $80 \%$ in the US-guided transplanted patient group and $45 \%$ in the control group $(p<0.5)$. No significant peri-procedural complications were observed. 


\section{Discussion}

In a well-functioning renal transplant patient, a dialysis fistula becomes useless and, moreover, may contribute to left ventricular hypertrophy. Stenosis is the most common complication of dialysis fistulas and the main cause of their thromboses. The exact percentage of fistulas with stenosis in renal transplant patients is unknown, but approximately half of all fistulas undergo thrombosis in these patients [4]. In these situations, renal graft insufficiency and restarting dialysis reveal problems with vascular access. It has been well documented that a dialysis fistula is the best vascular access. However, the Dialysis Outcomes and Practice Patterns Study (DOPPS) data show that patients returning to dialysis due to progressive kidney graft insufficiency more frequently use dialysis catheters than patients just starting dialysis [1]. There are some potential explanations for the higher rates of central venous catheter use among transplant failure patients. These include the fragmentation of care between the renal transplant and dialysis centres, an overemphasis on preservation of renal allograft function and an under-emphasis on pre-dialysis care, patient-induced delays including a reluctance to accept the need for dialysis and an unanticipated and rapid loss of kidney allograft function [7]. Among patients with native kidney function decline, multidisciplinary pre-dialysis care improves the use of surgical dialysis vascular access [1], and this kind of protection should be expanded to renal transplant patients. In many cases, proper functioning of a dysfunctional dialysis fistula may be restored with classical surgical techniques or endovascular procedures. Some reports have been published concerning redo operations, mainly of occluded dialysis fistulas in renal transplant patients returning to dialysis. Endovascular procedures are less invasive and are commonly used for treatment of vascular pathologies. Angioplasty is broadly used for the correction of dysfunctional dialysis fistulas. However, these interventions are routinely performed under angiographic control, which means exposure to $X$-rays and administration of contrast medium that may be potentially damaging to the kidneys, especially if their functioning is significantly impaired. In patients with advanced chronic kidney disease administration of a contrast agent for endovascular procedures can result in a relatively large renal injury, significant reduction in renal function and permanent end-stage renal disease (ESRD) [8]. In recent years, some articles have been published on the use of US as a method of imaging arteries and veins during intravascular procedures performed on dialysis fistulas [9-11]. The main advantage of this method is the lack of harmful influences on renal function, which is especially important for the preservation of remnant kidney function. Marks et al. described the use of duplex US to guide percutaneous interventions in a group of dialysis patients without additional fluoroscopy assistance [12]. Recently, Kumar et al. published a paper on the same subject, presenting a single-centre experience of 78 US-guided angioplasty procedures for treating stenoses of arteriovenous fistulas (AVFs) [13]. During a 24-month period of follow-up observations in the Kumar et al. study, $60.2 \%$ of dialysis fistulas had primary post-intervention patency after 12 months, $53.8 \%$ after 18 months and $48.9 \%$ after 24 months. Furthermore, this same group also reported secondary patency of $100 \%$ after 12 months, 95.4\% after 18 months and 89\% after 24 months.

Our primary and secondary patency percentages after 12 months were $80 \%$ and $90 \%$, respectively. Thus, these findings suggest very good angioplasty results performed under US guidance in renal transplant patients. Within our control group of patients after the standard angioplasty procedures, fistula thromboses and the need for repeated radiologic interventions due to restenosis were more common. Only $47 \%$ of those patients had primary patent fistulas 12 months after the original percutaneous transluminal angioplasty (PTA). In seven patients in this group, we performed redo procedures correcting fistula dysfunctions.

Differences between the study groups - concerning patient demographics and dialysis fistula characteristics - influence the results and should be taken into account when determining the conclusions. Angioplasty results are highly variable and can be influenced by several known clinical (e.g. newer fistula, older patients), anatomical (e.g. longer stenosis), biochemical and hemodynamic factors [14]. Outcome differences between groups in our study may have been caused by several factors. The mean age of our control group was significantly higher than our focus group (64 vs. 45 years, respectively), which is a known risk factor of shorter periods of patency. Additionally, the older age in the control group may have led to a significant mortality rate in 
these patients, specifically, $15 \%$ in the observation period over a mean of 20 months. In our opinion, the most significant difference between the groups that significantly influenced the results was variation in pre-operative fistula blood flows and stenosis severity. Differences in the dialysis fistula types, especially presence of a PTFE prosthesis, between groups might also have affected the results. Another factor affecting angioplasty outcomes in our US-guided group was immunosuppressive drugs. These agents (e.g. rapamycin) are inhibitors of myocyte migration and proliferation and can inhibit stenosis after vascular procedures. The small number of cases after transplantation $(n=10)$ in our study may also have biased our results.

In our study, there were no cases of any significant peri-procedural complications. However, US visualization for dialysis fistula angioplasty has some disadvantages. Gorin et al. reviewed 31 cases of US-guided angioplasty of autogenous AVFs and reported $6(11 \%)$ complications, two patients had proximal stenoses that could not be crossed under US guidance and there were four incidents of significant haematomas [15]. Difficulties with passing dialysis fistula stenoses with guidewires can be overcome by using fluoroscopy without contrast. Problems with imaging the proximal edge of a stenosis located in the subclavian or brachiocephalic veins can sometimes be reduced with special micro-convex probes, but these pathologies make it very difficult to obtain US visualization.

Another option for visualization of endovascular procedures without administration of nephrotoxic contrast agents is carbon dioxide $\left(\mathrm{CO}_{2}\right)$ angiography. Venous $\mathrm{CO}_{2}$ angiography can be performed anywhere in the chest and extremities, but because of the risk of neurotoxicity, intra-arterial $\mathrm{CO}_{2}$ angiography should not be performed above the diaphragm [16]. For this reason, $\mathrm{CO}_{2}$ visualization for endovascular procedures on an upper extremity dialysis fistula performed from femoral artery access is not more advantageous than ultrasound visualization. The only significant advantage of $\mathrm{CO}_{2}$ compared to ultrasound is better imaging of the subclavian, brachiocephalic and VCS veins. On the other hand, in our opinion, ultrasound visualization is more cost-effective, more accessible, and cheaper than $\mathrm{CO}_{2}$, while still providing good imaging of the dialysis fistula.

In our opinion, in many centres, a significant gap exists between transplant and dialysis unit care, and the loss of a dialysis fistula during transplant unit surveillance is quite common. There are no accepted guidelines for the management of dialysis fistulas in renal transplant recipients [17]. In our opinion, dialysis fistulas in renal transplant patients, especially with insufficient graft function, should be routinely examined with US and a precise physical examination. US-guided angioplasty of dialysis fistulas is a minimally invasive procedure that can be repeated without radiation hazards to either patients or physicians. Endovascular dilatation of a stenosis in a dialysis fistula diminishes the risk of fistula thrombosis [18], thus protecting its patency. Another benefit of US visualization over angiography-guided angioplasty is the lower cost of the procedure and the greater accessibility.

\section{Conclusions}

We have demonstrated, for the first time, the usefulness of minimally invasive corrections of dialysis fistulas in renal transplant patients performed with US guidance without contrast use. This is an effective and safe method. We think that US-guided angioplasty should be the method of choice for correction of detected stenoses of dialysis fistulas in patients with transplant kidney insufficiency. In our opinion, routine examinations of dialysis fistulas in renal transplant patients and percutaneous angioplasty of stenoses will decrease the number of patients re-entering dialysis with a dialysis catheter.

\section{Conflict of interest}

The authors declare no conflict of interest.

\section{References}

1. Perl J, Zhang J, Gillespie B, et al. Reduced survival and quality of life following return to dialysis after transplant failure: the dialysis outcomes and practice patterns study. Nephrol Dial Transplant 2012; 27: 4464-72.

2. Głowiński J, Małyszko J, Głowińska I, Myśliwiec M. To close or not to close: fistula ligation and cardiac function in kidney allograft recipients. Pol Arch Med Wewn 2012; 122: 348-52.

3. Grochowiecki T, Szmidt J, Gałązka Z, et al. Usefulness of arterialized cephalic vein of forearm of previously thrombosed arteiovenous fistula for creating a new vascular access for hemodialysis in patients with renal allograft insufficiency. Transplant Proc 2000; 3296: 1375-6.

4. Weyde W, Letachowicz W, Krajewska M, et al. Arteriovenous fistula reconstruction in patients with kidney allograft failure. Clin Transplant 2008; 22: 185-90. 
5. Bacchini G, Cappello A, La Milia V, et al. Color Doppler ultrasonography imaging to guide transluminal angioplasty of venous stenosis. Kidney Int 2000; 58: 1810-3.

6. DerDerian T, Hingorani A, Ascher E, et al. To BAM or not to BAM? A closer look at balloon-assisted maturation. Ann Vasc Surg 2013; 27: 104-9.

7. Gill JS, Abichandani R, Khan S, et al. Opportunities to improve the care of patients with kidney transplant failure. Kidney Int 2002; 61: 2193-200.

8. McCullough PA, Choi JP, Feghali GA, et al. Contrast-Induced acute kidney injury. J Am Coll Cardiol 2016; 68: 1465-73.

9. Ascher E, Hingorani A, Marks N. Duplex-guided balloon angioplasty of failing or nonmaturing arterio-venous fistulae for hemodialysis: a new office-based procedure. I Vasc Surg 2009; 50: 594-9.

10. Gallagher JJ, Boniscavage P, Ascher E, et al. Clinical experience with office-based duplex-guided balloon-assisted maturation of arteriovenous fistulas for hemodialysis. Ann Vasc Surg 2012; 26: 982-84

11. Wakabayashi M, Hanada S, Nakano H, et al. Ultrasound-guided endovascular treatment for vascular access malfunction: results in 4896 cases. J Vasc Access 2013; 14: 225-30.

12. Marks N, Ascher E, Hingorani AP. Duplex-guided repair of failing or nonmaturing arterio-venous access for hemodialysis. Perspect Vasc Surg Endovasc Ther 2007; 19: 50-5.

13. Kumar S, Mahajan S, Patl SS, et al. Ultrasound-guided angioplasty for treatment of peripheral stenosis of arteriovenous fistula - a single-center experience. J Vasc Access 2017; 18: 52-6.

14. Neuen BL, Gunnarsson R, Webster AC, et al. Predictors of patency after balloon angioplasty in hemodialysis fistulas: a systematic review. J Vasc Interv Radiol 2014; 25: 917-24.

15. Gorin DR, Perrino L, Potter DM, et al. Ultrasound-guided angioplasty of autogenous arteriovenous fistulas in the office setting. I Vasc Surg 2012; 55: 1701-5.

16. Sharafuddin MJ, Marjan AE. Current status of carbon dioxide angiography. J Vasc Surg 2017; 66: 618-37.

17. Manca O, Pisano GL, Carta P, et al. The management of hemodialysis arteriovenous fistulas in well unctioning renal transplanted patients: many doubts, few certainties. I Vasc Access 2005; 6: 182-6.

18. Ming Z, Li W, Ding W, et al. The efficacy of percutaneous transluminal angioplasty treatment for the patients with arteriovenous fistula dysfunction. Int Angiol 2016; 35: 163-9.

Received: 17.12.2018, accepted: 10.02.2019. 